\title{
GMR
}

\section{Identification of SNPs located on BTA 6 and BTA 20 significantly associated with bovine respiratory disease in crossbred cattle}

\author{
S.L. Miller ${ }^{1}$, S. Mizell ${ }^{1}$, R. Walker ${ }^{2}$, T. Page $^{1}$ and M.D. Garcia ${ }^{1,3}$ \\ ${ }^{1}$ School of Animal Sciences, Louisiana State University/LSU Agricultural Center, \\ Baton Rouge, LA, USA \\ ${ }^{2}$ LSU Agricultural Center Hill farm Research Station, Homer, LA, USA \\ ${ }^{3}$ Department of Animal, Dairy and Veterinary Sciences, Utah State University, \\ Logan, UT, USA \\ Corresponding author: M.D. Garcia \\ E-mail: Mgarcia768@aol.com / matthew.garcia@usu.edu
}

Genet. Mol. Res. 15 (4): gmr.15048861

Received June 7, 2016

Accepted July 29, 2016

Published October 6, 2016

DOI http://dx.doi.org/10.4238/gmr.15048861

Copyright (C) 2016 The Authors. This is an open-access article distributed under the terms of the Creative Commons Attribution ShareAlike (CC BY-SA) 4.0 License.

\begin{abstract}
The objective of the present study was to evaluate single nucleotide polymorphisms (SNPs) located in two quantitative trait locus (QTL) regions (BTA 6 and BTA 20) that are associated with bovine respiratory disease (BRD). A population of 323 crossbred steers sired by five purebred sire breeds during 2010-2013 (Angus, Braford, Braunvieh, Charolais, and Simmental) were evaluated for BRD susceptibility during the finishing process at a commercial feedlot. A total of 21 animals representing all sire breeds were affected with BRD at some time during the finishing process over the 4-year period. Although multiple sire breeds were evaluated in the present study, no sire breed effects were detected. A total of 82 SNPs were evaluated (58 on BTA 6 and 24 on BTA 20) in the present study for
\end{abstract}

Genetics and Molecular Research 15 (4): gmr.15048861 
potential associations with BRD incidence. When evaluating the previously described QTL regions on BTA 6, three SNPs (rs42968895, rs42823614, and rs43448463) were significantly $(\mathrm{P}<0.05)$ associated with BRD incidence. Another three SNPs (rs42477340, rs42512588, and rs42524468) were identified as significantly associated with BRD on the previously described BTA 6 QTL region. For both of these regions, animals inheriting different genotypes differed in BRD incidence during the finishing period. Although multiple SNPs were identified as being significantly associated with BRD incidence in the present study, these SNP associations should be validated in larger and more diverse populations.

Key words: SNP; Bovine respiratory disease; Feedlot; Finishing

\section{INTRODUCTION}

Bovine respiratory disease (BRD) is the most common disease affecting feedlot cattle. It contributes to approximately $75 \%$ of morbidity and $50-75 \%$ of mortality in feedlots annually (Brooks et al., 2011). Economic losses incurred by animals contracting BRD, due to reduced feed efficiency, veterinary treatment, and death, are estimated to cost the U.S. beef industry 640 million dollars annually (Bowland and Shewen, 2000). Although cattle of all ages can be affected by BRD, most are susceptible during times of high stress as young calves (Schneider et al., 2010). Commonly referred to as shipping fever, BRD is most prevalent in calves being transported to feedlots after being weaned (Bowland and Shewen, 2000). The severity of bovine respiratory infection has been linked to a variety of factors, including environmental and nutritional changes, transportation, and weaning factors. Of these factors, transportation is the most accepted non-infectious risk factor for BRD (Bowland and Shewen, 2000). Fatal respiratory infections occur when a primary viral infection compromises host defenses and enhances the severity of a secondary bacterial infection (Hodgson et al., 2005).

Identifying animals with genetic predisposition to BRD susceptibility/resistance is vitally important, as BRD is a lowly heritable trait (Snowder et al., 2005). As such, identification of single nucleotide polymorphisms (SNPs) significantly associated with BRD resistance has become of great importance to the beef industry (Dekkers, 2004). Previous studies have reported quantitative trait locus (QTL) regions associated with BRD susceptibility located on BTA 6 and BTA 20 (Li et al., 2004; Casas et al., 2010). Moreover, the U.S. meat and animal research center reported 30 SNPs on 15 chromosomes that were associated with BRD, including the previously described regions located on BTA 6 and BTA 20 (Casas et al., 2010). Thus, the objective of the present study was to evaluate SNPs located in the previously described QTL regions BTA 6 and BTA 20 for potential associations with BRD incidence in a population of crossbred steers finished in a commercial feedlot.

\section{MATERIAL AND METHODS}

All animals were treated and maintained in accordance with the principles and guidelines outlined in the Guide for the Care and Ethical Use of Agricultural Animals in Research and Teaching (Protocol \#AE2009-21). The animals utilized in the present study were

Genetics and Molecular Research 15 (4): gmr.15048861 
comprised of 323 crossbred steers born at the Louisiana State University Agricultural Center Central Research Station in Baton Rouge, LA and the Agricultural Center Hill Farm Research Station in Homer, LA from 2010 to 2013. Calves were born during the spring calving season and were managed at these facilities until weaning, or until approximately six to seven months of age. Calves were sired by Charolais, Braunvieh, Simmental, Angus, or Braford bulls. The dam breeds at the Central Research Station utilized for this study have been previously described during the characterization of the Germplasm Evaluation VIII studies (Wheeler et al., 2010). The dams utilized by the Hill Farm Research Station in Homer, LA comprised various breed backgrounds. Steers were given a preconditioning ration at weaning and were fed $2.24 \mathrm{~kg}$ per head or $1.5 \%$ of the calves' body weight each morning for 45 days. Along with feed, calves had access to pasture grazing and bales of hay. At weaning, the calves were vaccinated with Bova Shield Gold FP5 and Clostra Shield 7. Calves were then boostered with Bova Shield Gold FP5 and Clostra Shield 7 approximately 30 days later. Steers that were deemed healthy enough for shipping were vaccinated and shipped to commercial feedlots in Texas and Oklahoma for the finishing process. During the finishing process, animals were recorded as 0 if it was never affected with BRD and 1 if it was affected by BRD at some point during the finishing process. In this study, a total of 302 animals over the four-year period were recorded as unaffected by BRD, whereas 21 animals were affected with BRD (Table 1).

Table 1. Total number of animals for each sire breed and total number affected by BRD for each sire breed.

\begin{tabular}{l|c|c}
\hline Sire breed & Total No. of animals & \multicolumn{2}{c}{ Total No. affected by BRD } \\
\hline Angus & 55 & 4 \\
\hline Braford & 29 & 2 \\
\hline Braunvieh & 46 & 0 \\
\hline Charolais & 133 & 12 \\
\hline Simmental & 60 & 3 \\
\hline
\end{tabular}

Ear notches were collected from all calves at birth for future DNA extraction. Extraction of DNA was conducted using a saturated salt procedure previously described by Miller et al. (1988). DNA stock solutions were diluted to $25 \mathrm{ng} / \mu \mathrm{L}$ concentrations for future genotyping reactions. Fifty-eight SNPs were selected from a previously described QTL region associated with incidence of BRD, spanning between 40-80 Mbp on BTA 6 (Li et al., 2004). Another 24 SNPs were selected from a QTL region located on BTA 20 (0-30 Mbp) that has also been associated with BRD incidence (Casas et al., 2011). SNPs were selected using the QTL database (http://www.animalgenome.org/cgi-bin/QTLdb/ index). SNPs, allele substitutions, and upstream and downstream genomic sequences are presented in Tables 2 and 3. SNP genotyping was performed by Neogen, Inc. (Lincoln, NE, USA) using the Sequenom platform.

The mixed model procedure of SAS (v. 9.4, SAS Institute, Cary, NC, USA) was utilized to evaluate potential associations of SNPs located on BTA 6 and BTA 20 with BRD incidence. Only SNPs with more than one genotype were included in the analysis. The LSMEANS function, along with a pre-planned pairwise comparisons procedure, was utilized to evaluate if significant differences existed between individuals inheriting different SNP genotypes identified as significant for BRD incidence. The dependent variable in the model was incidence of BRD. The independent variables included sire breed, SNP genotype, and birth year. Sire breed (year) was fit into the model as a random nested variable to account for confounding effects of sire breeds among the four years. Significance in the model was set at $\mathrm{P} \leq 0.05$.

Genetics and Molecular Research 15 (4): gmr.15048861 
Table 2. Single nucleotide polymorphism ID, allele substitutions, and upstream and downstream genomic sequences utilized for amplification and visualization of genotypes for BTA 6 .

\begin{tabular}{|c|c|c|c|}
\hline$\overline{\text { SNP ID }}$ & Allele substitution & Forward sequence & Reverse sequence \\
\hline rs 29025265 & $\mathrm{C} / \mathrm{T}$ & CAGTTAGAGTTCAAAGGGACTTTTG & GTCAAACTGAGTACAAAATCTTTTC \\
\hline rs41626155 & $\mathrm{C} / \mathrm{T}$ & TCCTGCCCTGCCTTCTTTAACTTCT & TCCCCAATCTCTGGTTGCCATTCAT \\
\hline rs41653357 & $\mathrm{A} / \mathrm{C}$ & TGGAGAATCCTTTAGACAATAGGAG & TTGGTGGGCTATAGTCCATGGGGTT \\
\hline rs42402825 & $\mathrm{A} / \mathrm{G}$ & GAGAATCCAAAGACAATACCAAAAT & AAGTCTATTGAAAGCCCACTCCTTG \\
\hline rs42403565 & $\mathrm{C} / \mathrm{T}$ & ATTTCTATTACCCTATGTGTCAGAT & TCTGATTCACTCTTCTGCCTCCTCT \\
\hline rs42571566 & $\mathrm{A} / \mathrm{G}$ & GCCGTCTATGGGGTCGCACAGAGTC & GACACGACTGAAGCAACTTAGCAGC \\
\hline rs42579150 & $\mathrm{C} / \mathrm{T}$ & ATATGCCAATGATCTTAAAATTACT & GGTAAATATTTGAACATTTTTCTGC \\
\hline rs42579164 & $\mathrm{A} / \mathrm{C}$ & CTCTATTTTTACAACATGGATGGAC & TAGAGATGATTATACTAAGTGAAGA \\
\hline rs42725112 & $\mathrm{C} / \mathrm{T}$ & TTCATTAAAACACAAAAATCACAAC & AACTGCTGAACAACCACCAGCAAAA \\
\hline rs42823614 & $\mathrm{A} / \mathrm{C}$ & AGGCAAATTCTTCACCAGCTGAACC & CAGGGAAAGCCTAATTCCCACCTTC \\
\hline rs42824344 & $\mathrm{C} / \mathrm{T}$ & GTAGCATCATTGCCCTTTAATTATC & AAACTAGAAGCAAACTGAATGTCCA \\
\hline rs42880470 & $\mathrm{A} / \mathrm{G}$ & TCTGGAGTAGGTACTGTGGGAGCAA & CTCAATCAGAGTTGTGAATAGCCTC \\
\hline rs42880522 & $\mathrm{A} / \mathrm{G}$ & CTGAGGCTGGCCCTGACCTGAGATA & CСАСССТТTCТTАСТСТСТТТСТTC \\
\hline rs42900120 & $\mathrm{G} / \mathrm{T}$ & GGGGAAGGGGAGGAAGGATAAATTG & GAGATTGGGACTGACATATACACAC \\
\hline rs42900130 & $\mathrm{A} / \mathrm{G}$ & CACAGGAGATAATCCTCTGCCTCCA & TTATGGTCTTCTGTGAAAAGTACTG \\
\hline rs42900481 & $\mathrm{A} / \mathrm{G}$ & ACTTTAGATTCAATTCTTCTTGGCT & GGGATGGAGAATCTTTGAATTTCTC \\
\hline rs42961863 & $\mathrm{C} / \mathrm{T}$ & GGACCAGAAGTCCCTTTCCCTTGCT & ATGTGTATTTTTAATGGTGATGACA \\
\hline rs42961866 & $\mathrm{G} / \mathrm{T}$ & TGTTGTTTCCAGCTCTCCAATCTAG & TATTGTCCATTACTATTAAACATTC \\
\hline rs42961882 & $\mathrm{A} / \mathrm{T}$ & CTTCTTTTTTGGTATGATTTTGGTC & CTGTCTCCTATACACTATTACAGAT \\
\hline rs42968197 & $\mathrm{C} / \mathrm{T}$ & TGACAAGTAGATGCTTTTTATTAAA & TCATTCTATGTAAGAGACAGCTGAG \\
\hline rs42968891 & $\mathrm{A} / \mathrm{G}$ & TTCATACCTAGATAATTGCAATTTC & TACCTAGCCTTTCCAGTCCTTTGGA \\
\hline rs42968895 & $\mathrm{C} / \mathrm{T}$ & TCATATTCAGAGGTGGGATGTCATT & TTAAGGCTTTCAAGGCACTAATCCT \\
\hline rs43089863 & $\mathrm{C} / \mathrm{G}$ & CATCTCTCTGAGTTGTCCTCTATTG & AGTCAGGGAGCAGGGCCTTTTTACC \\
\hline rs43138398 & $\mathrm{C} / \mathrm{T}$ & TTGCAAGATAATTACAGTCACTTCC & TTTTCATGATCATTGGCCTTGAGCT \\
\hline rs43194943 & $\mathrm{A} / \mathrm{G}$ & ATATCTTCTTAATATCTTCTTTTTT & TTAGGTCTGCACCATTTCTGTCCTT \\
\hline rs43446022 & $\mathrm{G} / \mathrm{C}$ & TATGTTCAGAGGAATTAAGTCTTGA & CTTGTCATAAATACAACAAAATGAG \\
\hline rs43446601 & $\mathrm{G} / \mathrm{T}$ & GTTTCCTGGAATTTGGATGAAAATT & CCTTCAATGTTTATATCTGAATCTT \\
\hline rs43446955 & $\mathrm{C} / \mathrm{T}$ & TGCTTGTTTATATCACTTTGATATA & ACTATATTAAATTATAATGCTCTTT \\
\hline rs43447179 & $\mathrm{A} / \mathrm{G}$ & TTTCTTTTTTCCCACCAGGAAATAC & CATTTCCTGGCCTCATAAAGACCAT \\
\hline rs43448463 & $\mathrm{A} / \mathrm{G}$ & AGAATGCAAAGAGGAACTAAAGAGC & TCTTGATGAGGTTGAAGGAGAAGAA \\
\hline rs43448512 & $\mathrm{A} / \mathrm{C}$ & AGATAAACTGAGACTTTCATGACGG & AGGCTCTTGAAGGAGAAGTTCTTTG \\
\hline rs43449040 & $\mathrm{A} / \mathrm{G}$ & CACATTGATCGCTCTAATCTTAGAG & AAAAGTGCTTAAAAACTTAGACACT \\
\hline rs43449194 & $\mathrm{C} / \mathrm{T}$ & TGAAAATGTTTCTTGCATTATTTTA & TATCAATTTCTTCATTTTGCTGTTA \\
\hline rs43449209 & $\mathrm{A} / \mathrm{G}$ & AGTTGCTCAAGATCACACAGCATGT & TGCTGGAGCTAGGATTGAAAGCTCA \\
\hline rs43449835 & $\mathrm{C} / \mathrm{T}$ & TAGTATCCTTTGCTAAATTTATCAT & AGTAGGTTAAAGAAGCCTTCAGGAT \\
\hline rs43449896 & $\mathrm{A} / \mathrm{C}$ & TCCACTGGATGATCCACTGGATCAT & GAAAAAGCAAGAGAGTTCAAGAAAA \\
\hline rs43451134 & $\mathrm{A} / \mathrm{T}$ & CATACTATATAGCACAGGAAACTAT & TTCAATATCCTGGGATAAATCATAA \\
\hline rs42403543 & $\mathrm{C} / \mathrm{T}$ & AAGGAAATGCTTTCAATTTTTCACT & TTTATTATGATGCAAGCTGAAGGTT \\
\hline rs42481129 & $\mathrm{A} / \mathrm{G}$ & TTCTCCCACACCACAGTTTAAAAGC & TCAATTCTTCGGCACTCTGCCTTCT \\
\hline rs42579148 & $\mathrm{G} / \mathrm{T}$ & TATGACTTACCTACTGCTTTTCTTT & TATCTATGATGTCATAGAATGTAAG \\
\hline rs42823610 & $\mathrm{C} / \mathrm{T}$ & GCCATCCAGCCATCTCATCCTCTGT & GTCCCCTTCTCCTCCTGCCCCCAAT \\
\hline rs42824331 & $\mathrm{A} / \mathrm{G}$ & CATGGGGTCGCTGAGGGTCAGACAC & ACTGAGTGACTTCACTTTCACTTTT \\
\hline rs42725042 & $\mathrm{G} / \mathrm{T}$ & AGGGGAGAAGGGGACGATAGAGGAT & AGATGGCTGGATGGCATCACTGACT \\
\hline rs43080446 & $\mathrm{G} / \mathrm{T}$ & TTAAAGGAAAGATTACTTTATACAA & TATAAAGTATTGAAACAATAGTCTA \\
\hline rs43185776 & $\mathrm{C} / \mathrm{G}$ & TCCTATGTCATCCCCTTCTCCTCCT & CCCTCAATCCCTCCCAGCATCAGAG \\
\hline rs43178720 & $\mathrm{A} / \mathrm{T}$ & TGTATGTCTGTATGTACAGACATAC & GTGAAATATGTATATATGTACAGAC \\
\hline rs43449906 & $\mathrm{G} / \mathrm{T}$ & TATATAAAATTGCATTTTAGAAAAC & TAAAGGTGATTAATGCTTTTTAATT \\
\hline rs43449868 & $\mathrm{A} / \mathrm{G}$ & CCTAGAGCCAGACATCCTGGAATGC & AAGTCAAGTGGGCCTTAGGAAGCAT \\
\hline rs43448433 & $\mathrm{A} / \mathrm{G}$ & ATTGAAGAATCTCTTTCTATATTCT & AATATTCTTAGTTTTCACATCCCCC \\
\hline rs42940872 & $\mathrm{C} / \mathrm{G}$ & ATACAGCCAAAGGCTTTAGCAAAGT & ATGAAGCAGAAGTGTATGATTTTCT \\
\hline rs43130086 & $\mathrm{A} / \mathrm{G}$ & AACTTAGGTGAGCTGAGGGGGCTGA & GGAAATCCACACAAGTCGCCCATGA \\
\hline rs43444877 & $\mathrm{A} / \mathrm{G}$ & TCTGAAGAGTTCTTATCCCAAGAAA & AAAATTTTTTTTCTATTTCTTTAAT \\
\hline rs43445941 & $\mathrm{G} / \mathrm{T}$ & AAACTCCAATACTTTGACCACCTGA & GCAAAGAACTGACTCATTAGAAAAA \\
\hline rs43445971 & $\mathrm{A} / \mathrm{G}$ & TACATTTAGAACTGCTTACTTTCAT & TAAGTTCTTATGTAACACATAGATT \\
\hline rs42900433 & $\mathrm{G} / \mathrm{T}$ & CTGACTCTTGGCGATCCCATGGACT & TAGCATACCAGGCTCCTCTGTCCAT \\
\hline rs42961881 & $\mathrm{C} / \mathrm{T}$ & TGAATGCAACACTTTAACAGCATCA & CTTTAGTATTTGAAATAGCTCAGCT \\
\hline rs42725037 & $\mathrm{A} / \mathrm{G}$ & ATATATGTTCCTTAAGAAACAAAAA & TAGACCTACCATATGTAATCTTGCA \\
\hline rs43452444 & $\mathrm{C} / \mathrm{T}$ & AAGAAAAGGCAGTGTGCAAACAGGG & GTGAGCCACGTGAGAGAGAAGGTCG \\
\hline
\end{tabular}

Genetics and Molecular Research 15 (4): gmr.15048861 
Table 3. Single nucleotide polymorphisms ID, allele substitutions, and upstream and downstream genomic sequences utilized for amplification and visualization of genotypes for BTA 20.

\begin{tabular}{|c|c|c|c|}
\hline SNP ID & Allele substitution & Forward sequence & Reverse sequence \\
\hline rs41595713 & $\mathrm{C} / \mathrm{T}$ & TCTCGGTTCCTAACACAGCCAAGAC & GTTGTCCCGAACGGGTGAGGAATGG \\
\hline rs41931108 & $\mathrm{C} / \mathrm{G}$ & TTGGTGTGCCAAGCACATCCCCAGC & GAGGAAGGCAGGTTGTGCCCATATT \\
\hline rs42476237 & $\mathrm{C} / \mathrm{T}$ & CCTGGCCCACCCTTCCTTCCTTCCC & ATTTGTGGAGAAGCACGTGGGGAAC \\
\hline rs42476290 & $\mathrm{A} / \mathrm{G}$ & CTGAGGCCAGAATTCTTGAAAGAAT & TGTTTGCATGGTGACAGCAAAGCAT \\
\hline rs42477340 & $\mathrm{C} / \mathrm{T}$ & CTCCCGCCTCCTTCTCTGCTCCCTC & GGCTCCCTCTCTGCTCCCTCCGGCT \\
\hline rs42480445 & $\mathrm{C} / \mathrm{T}$ & TGGCCCCAAATGCCAAAAGGTTATC & TCATTTTTTTCCAAGCAATCCCACC \\
\hline rs42481107 & $\mathrm{A} / \mathrm{G}$ & AACAACACCTTCCACCGCCCCATCC & GGTCTCAGCCTAAGCATCAGCTCTT \\
\hline rs42512588 & $\mathrm{C} / \mathrm{T}$ & GAATGGGGAGTGACTGCTTCCTAGG & CTGGGGTTTCTGTTGGGTTGATGCT \\
\hline rs42520493 & $\mathrm{A} / \mathrm{C}$ & CATGCTGTATATCGGAGGGTCTAGG & CTGTTAAGCAGGAAATGAGAACTCC \\
\hline rs42524445 & $\mathrm{C} / \mathrm{T}$ & GGTTCTTAAAAGTGAAATGATAATG & AGAAGAAATAGAGTGATGTGATGTG \\
\hline rs 42524450 & $\mathrm{C} / \mathrm{T}$ & TCCTTGGAAGTGGGGTTGCTCCTTC & GGCCGCCACCCCTGGCCTCAGGCGT \\
\hline rs42524466 & $\mathrm{C} / \mathrm{G}$ & ATTTTATGTCGCAGTTTTCTCTCAC & AATCTAAGTTTAAATCTCTCAGAGG \\
\hline rs42524468 & $\mathrm{A} / \mathrm{T}$ & AATAGACCCACAGACATAGAAAACA & ATGTATGGTTACCAAAGGGGAAAGG \\
\hline rs42524472 & $\mathrm{A} / \mathrm{T}$ & AAAATAAATAGTAAATCACAAACAC & AATCACAGATAGGAAGAAAATGCAA \\
\hline rs42524503 & $\mathrm{A} / \mathrm{T}$ & GTTGTATAGACAGATATCTGTCACT & ATTCTTTCCAAATGCTCTGACAGAT \\
\hline rs43036576 & $\mathrm{A} / \mathrm{G}$ & TAATCATGAAGCCATCCTGTAGGGT & GAGCTAGGGTTTATAGCGGCTGTGA \\
\hline rs42524459 & $\mathrm{C} / \mathrm{T}$ & ATCCACACAGTCAAAGCCTTTGGCA & AGTCAATAAAGCAGAAATAGATGTT \\
\hline rs 42481060 & $\mathrm{C} / \mathrm{T}$ & ACTGCCTCAGGCCTGGCACACAGCC & GAGAGGCCATGGGGCCCTGTGGAGC \\
\hline rs41931083 & $\mathrm{C} / \mathrm{T}$ & GACTTCATTTCTCTCCGTGATAATC & TGCGGGGCAGGTCCCCAGGTCTGGA \\
\hline rs42524449 & $\mathrm{A} / \mathrm{G}$ & TCTGCCCCTGCTGACCTTCAACGTG & AATAGCTCCTCTAGGACCTCCTGCG \\
\hline rs42524457 & $\mathrm{G} / \mathrm{T}$ & GTTTATTGTGATCCACACAGTCAAA & CCTTTGGCACAGTCAATAAAGCAGA \\
\hline rs42476309 & $\mathrm{C} / \mathrm{T}$ & GATGGTTTAGTCACTAAGTCATGTC & GACTCTTGAAACCCCATGGACTGTA \\
\hline rs42236701 & $\mathrm{A} / \mathrm{C} / \mathrm{G}$ & TCCACTTGATTTCACATTCCAGGAT & TCTGGCTCTAGGTGAGTGATCACAC \\
\hline rs41931859 & $\mathrm{C} / \mathrm{T}$ & GAGGAGCCTGGGCTACAGTTCATGG & GTCACAGAGAGTCGGACACAACTGA \\
\hline
\end{tabular}

\section{RESULTS}

The analyses of BRD incidence revealed six significant individual SNPs. Three of the SNPs that were significant for BRD incidence were located on each of BTA 6 (rs42823614, rs42968895, and rs43448463) and BTA 20 (rs42477340, rs42512588, and rs42524468) (Table 4). Animals inheriting differing genotypes for each of the significantly identified SNP's had differing levels of BRD susceptibility or resistance (Table 5). Although multiple SNPs were described as significant sire breed was not identified as a significant source of variation, thus significance associations can be attributed to individual SNP and not sire breed.

Table 4. Level of significance and frequency of animals from each genotype associated with incidence of bovine respiratory disease.

\begin{tabular}{l|c|c|c|c|c|c|c}
\hline BTA & SNP ID & Allele & $\begin{array}{c}\text { Minor genotype } \\
\text { frequency }\end{array}$ & $\begin{array}{c}\text { Heterozygous genotype } \\
\text { frequency }\end{array}$ & $\begin{array}{c}\text { Major genotype } \\
\text { frequency }\end{array}$ & $\begin{array}{c}\text { SNP P } \\
\text { value }\end{array}$ & $\begin{array}{c}\text { Sire breed P } \\
\text { value }\end{array}$ \\
\hline 6 & $\mathrm{rs} 42823614$ & $\mathrm{~A} / \mathrm{C}$ & 3 & 50 & 253 & 0.0396 & 0.9339 \\
\hline 6 & $\mathrm{rs} 42968895$ & $\mathrm{C} / \mathrm{T}$ & 50 & 168 & 88 & 0.0234 & 0.9673 \\
\hline 6 & $\mathrm{rs} 43448463$ & $\mathrm{~A} / \mathrm{G}$ & 23 & 92 & 155 & 0.0334 & 0.9699 \\
\hline 20 & $\mathrm{rs} 42477340$ & $\mathrm{C} / \mathrm{T}$ & 114 & 52 & 116 & 0.0192 & 0.9906 \\
\hline 20 & $\mathrm{rs} 42512588$ & $\mathrm{C} / \mathrm{T}$ & 48 & 154 & 104 & 0.0167 & 0.9924 \\
\hline 20 & $\mathrm{rs} 42524468$ & $\mathrm{~A} / \mathrm{T}$ & 21 & 73 & 180 & 0.0324 & 0.6240 \\
\hline
\end{tabular}

${ }^{1}$ The major allele is presented on the left.

Genetics and Molecular Research 15 (4): gmr.15048861 
Table 5. Single nucleotide polymorphisms associated with bovine respiratory disease and least square means estimate comparisons between reported genotypes for incidence of bovine respiratory disease.

\begin{tabular}{l|c|c|c|c|c|c}
\hline Trait & BTA & SNP ID & Allele & Major genotype mean & Heterozygous genotype mean & Minor genotype mean \\
\hline BRD $^{1}$ & 6 & rs42823614 & A/C & $0.071 \pm 0.059^{\mathrm{a}}$ & $0.179 \pm 0.071^{\mathrm{b}}$ & $0.033 \pm 0.157^{\mathrm{ab}}$ \\
\hline BRD & 6 & $\mathrm{rs} 42968895$ & $\mathrm{C} / \mathrm{T}$ & $0.028 \pm 0.061^{\mathrm{a}}$ & $0.121 \pm 0.058^{\mathrm{bc}}$ & $0.057 \pm 0.065 \mathrm{a}^{\mathrm{ac}}$ \\
\hline BRD & 6 & $\mathrm{rs} 43448463$ & $\mathrm{~A} / \mathrm{G}$ & $0.061 \pm 0.050^{\mathrm{a}}$ & $0.090 \pm 0.053^{\mathrm{a}}$ & $0.232 \pm 0.074^{\mathrm{b}}$ \\
\hline BRD & 20 & $\mathrm{rs} 42477340$ & $\mathrm{C} / \mathrm{T}$ & $0.093 \pm 0.062^{\mathrm{ab}}$ & $0.162 \pm 0.068^{\mathrm{a}}$ & $0.030 \pm 0.063^{\mathrm{b}}$ \\
\hline BRD & 20 & $\mathrm{rs} 42512588$ & $\mathrm{C} / \mathrm{T}$ & $0.047 \pm 0.058^{\mathrm{a}}$ & $0.072 \pm 0.055^{\mathrm{a}}$ & $0.184 \pm 0.064^{\mathrm{b}}$ \\
\hline BRD & 20 & $\mathrm{rs} 42524468$ & $\mathrm{~A} / \mathrm{T}$ & $0.119 \pm 0.037^{\mathrm{a}}$ & $0.017 \pm 0.046^{\mathrm{b}}$ & $0.025 \pm 0.074^{\mathrm{b}}$ \\
\hline
\end{tabular}

Different superscripted letters indicate significant differences of means at $\mathrm{P} \leq 0.05$ within rows. ${ }^{1} \mathrm{BRD}=\mathrm{Bovine}$ respiratory disease. ${ }^{2}$ The major allele is presented on the left.

\section{DISCUSSION}

Three of the SNPs identified in the present study that were located on BTA 6 were significantly associated with BRD incidence. These results are in agreement with previous research that identified a significant QTL region along with significant SNPs associated with BRD incidence located on BTA 6 (Li et al., 2004). However, BRD was not the only economically important trait to be significantly associated with this region on BTA 6 . Growth traits such as birth weight, weaning weight (Lu et al., 2013a), hip height (Bolormaa et al., 2014), and carcass traits, such as carcass weight (Lu et al., 2013b), rib eye area (Casas et al., 2000), marbling (Lee et al., 2012), and back fat thickness (Li et al., 2004), have also been found to be significantly associated with this region.

Furthermore, three identified SNPs located on BTA 20 were also associated with BRD incidence. These results are in agreement with a previous study that identified SNPs significantly associated with incidence of BRD on BTA 20 (Casas et al., 2010). Like the BTA 6 region, BTA 20 has also been associated with other economically important traits such as the growth traits birth weight, weaning weight (Saatchi et al., 2014), and hip height (Bolormaa et al., 2014), and carcass traits such as carcass weight (McClure et al., 2010), yield grade, marbling (Saatchi et al., 2014), rib eye area (Garcia et al., 2010), and back fat thickness (Garrett et al., 2008).

These results indicate that BRD susceptibility may have a strong genetic component in conjunction with an environmental influence. To validate the results of the present study, a larger, more diverse population of animals should be utilized. Additional SNPs located on BTA 6 and BTA 20, as well as evaluation of SNPs in other regions of the bovine genome, should be evaluated. This will ensure that SNPs accounting for the most variation in BRD susceptibility are identified, which will lead to improved accuracy for identifying genetic predisposition to BRD. Furthermore, the identified SNPs in these regions should be evaluated for multiple traits so that selection for specific SNPs associated with BRD does not result in detrimental selection of other economically important traits.

\section{Conflicts of interest}

The authors declare no conflict of interest.

\section{ACKNOWLEDGMENTS}

Research supported by the Louisiana Agricultural Experiment Station through the use of State and Federal Hatch funds. 


\section{REFERENCES}

Bolormaa S, Pryce JE, Reverter A, Zhang Y, et al. (2014). A multi-trait, meta-analysis for detecting pleiotropic polymorphisms for stature, fatness and reproduction in beef cattle. PLoS Genet. 10: e1004198. http://dx.doi. org/10.1371/journal.pgen.1004198

Bowland SL and Shewen PE (2000). Bovine respiratory disease: commercial vaccines currently available in Canada. Can. Vet. J. 41: 33-48.

Brooks KR, Raper KC, Ward CE, Holland BP, et al. (2011). Economic effects of bovine respiratory disease on feedlot cattle during backgrounding and finishing phases. Prof. Anim. Sci. 27: 195-203. http://dx.doi.org/10.15232/S10807446(15)30474-5

Casas E, Shackelford SD, Keele JW, Stone RT, et al. (2000). Quantitative trait loci affecting growth and carcass composition of cattle segregating alternate forms of myostatin. J. Anim. Sci. 78: 560-569. http://dx.doi.org/10.2527/2000.783560x

Casas E, Kuehn L, Snelling W and Wells J (2010). Genomics of disease in beef cattle. Proceedings of the 9th World Congress on Genetics Applied to Livestock Production, Leipzig, Germany. August 1-6, 2010. CD-ROM Communication No. 0125.

Casas E, Garcia MD, Wells JE and Smith TP (2011). Association of single nucleotide polymorphisms in the ANKRA2 and CD180 genes with bovine respiratory disease and presence of Mycobacterium avium subsp. paratuberculosis(1). Anim. Genet. 42: 571-577. http://dx.doi.org/10.1111/j.1365-2052.2011.02189.x

Dekkers JC (2004). Commercial application of marker- and gene-assisted selection in livestock: strategies and lessons. $J$. Anim. Sci. 82 (E-Suppl): E313-E328.

Garcia MD, Thallman RM, Wheeler TL, Shackelford SD, et al. (2010). Effect of bovine respiratory disease and overall pathogenic disease incidence on carcass traits. J. Anim. Sci. 88: 491-496. http://dx.doi.org/10.2527/jas.2009-1874

Garrett AJ, Rincon G, Medrano JF, Elzo MA, et al. (2008). Promoter region of the bovine growth hormone receptor gene: single nucleotide polymorphism discovery in cattle and association with performance in Brangus bulls. J. Anim. Sci. 86: 3315-3323. http://dx.doi.org/10.2527/jas.2008-0990

Hodgson PD, Aich P, Manuja A, Hokamp K, et al. (2005). Effect of stress on viral-bacterial synergy in bovine respiratory disease: novel mechanisms to regulate inflammation. Comp. Funct. Genomics 6: 244-250. http://dx.doi.org/10.1002/ $\underline{\operatorname{cfg} .474}$

Lee JH, Li Y and Kim JJ (2012). Detection of QTL for carcass quality on chromosome 6 by exploiting linkage and linkage disequilibrium in Hanwoo. Asian-australas. J. Anim. Sci. 25: 17-21. http://dx.doi.org/10.5713/ajas.2011.11337

Li C, Basarab J, Snelling WM, Benkel B, et al. (2004). Identification and fine mapping of quantitative trait loci for backfat on bovine chromosomes 2, 5, 6, 19, 21, and 23 in a commercial line of Bos taurus. J. Anim. Sci. 82: 967-972. http:// dx.doi.org/10.2527/2004.824967x

Lu D, Sargolzaei M, Kelly M, Vander Voort G, et al. (2013a). Genome-wide association analyses for carcass quality in crossbred beef cattle. BMC Genet. 14: 80. http://dx.doi.org/10.1186/1471-2156-14-80

Lu D, Miller S, Sargolzaei M, Kelly M, et al. (2013b). Genome-wide association analyses for growth and feed efficiency traits in beef cattle. J. Anim. Sci. 91: 3612-3633. http://dx.doi.org/10.2527/jas.2012-5716

McClure MC, Morsci NS, Schnabel RD, Kim JW, et al. (2010). A genome scan for quantitative trait loci influencing carcass, post-natal growth and reproductive traits in commercial Angus cattle. Anim. Genet. 41: 597-607. http:// dx.doi.org/10.1111/j.1365-2052.2010.02063.x

Miller SA, Dykes DD and Polesky HF (1988). A simple salting out procedure for extracting DNA from human nucleated cells. Nucleic Acids Res. 16: 1215. http://dx.doi.org/10.1093/nar/16.3.1215

Saatchi M, Schnabel RD, Taylor JF and Garrick DJ (2014). Large-effect pleiotropic or closely linked QTL segregate within and across ten US cattle breeds. BMC Genomics 15: 442. http://dx.doi.org/10.1186/1471-2164-15-442

Schneider MJ, Tait RG, Jr., Ruble MV, Busby WD, et al. (2010). Evaluation of fixed sources of variation and estimation of genetic parameters for incidence of bovine respiratory disease in preweaned calves and feedlot cattle. J. Anim. Sci. 88: 1220-1228. http://dx.doi.org/10.2527/jas.2008-1755

Snowder GD, Van Vleck LD, Cundiff LV and Bennett GL (2005). Influence of breed, heterozygosity, and disease incidence on estimates of variance components of respiratory disease in preweaned beef calves. J. Anim. Sci. 83: 1247-1261. http://dx.doi.org/10.2527/2005.8361247x

Wheeler TL, Cundiff LV, Shackelford SD and Koohmaraie M (2010). Characterization of biological types of cattle (Cycle VIII): carcass, yield, and longissimus palatability traits. J. Anim. Sci. 88: 3070-3083. http://dx.doi.org/10.2527/ jas.2009-2497

Genetics and Molecular Research 15 (4): gmr.15048861 